\title{
Interventions to modify physical activity in patients with COPD: where do we go from here?
}

\author{
Daniel Langer ${ }^{1,2}$ and Heleen Demeyer ${ }^{1,2,3}$
}

Affiliations: ${ }^{1} \mathrm{KU}$ Leuven-University of Leuven, Faculty of Kinesiology and Rehabilitation Sciences, Leuven, Belgium. ${ }^{2}$ University Hospitals Leuven, Respiratory Rehabilitation and Respiratory Division, Leuven, Belgium. ${ }^{3}$ Center for Research in Environmental Epidemiology (CREAL), Barcelona, Spain.

Correspondence: Daniel Langer, Laboratory of Respiratory Diseases, KU Leuven - Campus Gasthuisberg O\&N1, Herestraat 49 box 706, B-3000, Leuven, Belgium. E-mail: daniel.langerlakuleuven.be

@ERSpublications

More research into coaching interventions for improving participation in physical activity in COPD patients is needed http://ow.ly/4neMkA

Assessing participation in physical activity as an outcome in patients with chronic obstructive pulmonary disease (COPD) has received growing attention in recent years $[1,2]$. This can be explained both by an increased recognition of health effects associated with physical (in)activity and by the high prevalence of physical inactivity in patients suffering from COPD. Benefits of physical activity in the general population and in the elderly are well documented and include a reduction in cardiovascular disease, type 2 diabetes, cancer, dementia, risk of falling and all-cause mortality [3-5]. Physical activity has also been associated with better preservation of bone mass, higher quality of life and improved cognitive function $[4,5]$. Interestingly the protective effects of (increases in) physical activity on (cardiovascular) morbidity and mortality have been shown to be present independent of physical fitness levels [6]. Inactivity has been shown to be highly prevalent in patients with COPD [7]. Duration of activities has been shown to be slightly more reduced than the intensity of activities $(57 \%$ and $75 \%$, respectively, of values observed in healthy controls) [8]. A reduction in physical activity is already present early in the disease and worsens with progressing disease severity $[9,10]$. Even (ex-)smokers unaware of their airflow limitation show reductions in physical activity when compared with (ex-)smokers without airflow limitation [11]. When compared with other chronic conditions, such as diabetes and rheumatoid arthritis, inactivity is more prevalent in patients with COPD [12]. The association of physical activity with health outcomes specifically relevant to patients with COPD has also been studied in recent years. It has been demonstrated that increased participation in physical activity is associated with reduced risks for hospital admission and mortality [13]. From data collected in preclinical patients with COPD (i.e. (ex-)smokers unaware of their airflow limitation) it was concluded that inactivity and smoking, rather than the disease COPD as such, were the main risk factors for developing comorbid conditions (cardiovascular, metabolic and musculoskeletal disease) [14]. Patients progressively decrease physical activity over time [15]. Some data are also available on the impact of changes in activity behaviour on health status. EsTEBAN et al. [16] showed that patients who increased their physical activity over a 2 year follow-up showed a decreased rate of exacerbations requiring hospitalisation in the next 3 years. Patients with COPD who gradually decreased their activity levels increased their mortality risk and showed a faster disease progression [15, 17]. These associations of physical activity with health outcomes, along with the high prevalence of inactivity in patients with COPD, have led to an increased interest in interventions to increase participation in physical activity in this population.

Received: April 152016 | Accepted: April 272016

Support statement: D. Langer is a postdoctoral fellow of Research Foundation Flanders. H. Demeyer is the recipient of a joint ERS/SEPAR Fellowship (LTRF 2015).

Conflict of interest: None declared.

Copyright OERS 2016 
In this issue of the European Respiratory Journal, MANTOANI et al. [18] report a systematic review of 60 interventional studies that have assessed physical activity as an outcome in patients with COPD. Seven intervention groups were identified, including counselling interventions, pulmonary rehabilitation programmes and pharmacological treatment with bronchodilators. The authors conclude that counselling programmes, especially when combined with coaching interventions, and longer lasting ( $>12$ weeks) pulmonary rehabilitation programmes have the greatest potential to modify physical activity behaviour. Other interventions described in the review by MANTOANI et al. [18] such as dietary interventions, oxygen therapy, bronchodilators, noninvasive ventilation and neuromuscular electrical stimulation should probably not be regarded as effective stand-alone interventions. These therapies can be used as adjuncts to counselling and rehabilitation interventions to reduce symptoms and facilitate engagement in physical activity. The overall quality of the included studies was low, and therefore, the relative benefit of different interventions on physical activity levels is still debatable. Based on the data presented by MANTOANI et al. [18] and our own experience some suggestions can be provided for future research priorities in this area. MANTOANI et al. [18] reported small average improvements in physical activity after pulmonary rehabilitation programmes, with slightly larger changes observed after longer lasting programmes. These findings are in line with results from cardiac and orthopaedic rehabilitation programmes. In these populations it has also been shown that most patients failed to maintain an active lifestyle after acutely increasing their exposure to structured and planned physical activity (i.e. supervised exercise training) during a rehabilitation programme [19]. This is not surprising, since the focus during these rehabilitation programmes is mainly on increasing functional exercise capacity and improving symptoms and not on improving physical activity. Rehabilitation programmes do not usually include specific elements designed for modifying physical activity behaviour in daily life outside of the rehabilitation setting. This probably explains why the physical activity counselling interventions reviewed by MANTOANi et al. [18] were more consistently successful in modifying physical activity than rehabilitation programmes. The effectiveness of counselling interventions to change physical activity behaviour has been shown to be causally linked to mobilising social support and the use of established behaviour change strategies. These include self-monitoring, stimulus control, problem solving, relapse prevention management, goal setting, self-reinforcement and developing action plans [20-22]. It has also been suggested to assess not only the actual behaviour (i.e. participation in physical activity), but also readiness and motivation to change during these interventions [21]. These recommendations are consistent with recent guidelines from the American Heart Association promoting the use of motivational interviewing as well as goal-setting, self-monitoring and a high contact frequency in interventions to promote regular physical activity [23].

Wearable monitors providing feedback to users have been used as a treatment component in numerous physical activity counselling interventions. These home-based pedometer walking programmes were successful in getting sedentary adults to walk 2000 steps $(\sim 30 \%)$ more per day [24]. The effects of counselling interventions included in the review by MANTOANI et al. [18] resulted in average increases in comparison to control groups ranging from 600 to 3000 steps ( 20\%). We have recently observed in our centre that the likelihood of achieving an improvement in physical activity of at least 1000 steps per day, in response to participation in a pulmonary rehabilitation programme, increased gradually from $18 \%$ in patients with severely reduced functional exercise capacity $(6-\mathrm{min}$ walking distance $(6 \mathrm{MWD})<300 \mathrm{~m})$ to $52 \%$ in patients with better preserved functional capacity $(6 \mathrm{MWD}>450 \mathrm{~m})$ [25]. We also observed that a physical activity coaching intervention was not effective in patients recruited immediately after an exacerbation-related hospital admission [26]. Patients with better preserved levels of functional exercise capacity are therefore probably good candidates for physical activity coaching programmes. Patients with more severe reductions in functional capacity should probably first be referred to a pulmonary rehabilitation programme to increase their functional reserve. Integrating components of physical activity coaching interventions into pulmonary rehabilitation programmes might also be an attractive option for these patients. Two randomised controlled trials published after the final inclusion date for the review by MANTOANi et al. [18] have studied this combined approach [26-28]. Cruz et al. [28] observed an increase in physical activity, while no additional effects of the combined intervention were observed in the study by BurTin et al. [27]. Differences in the characteristics of the included patients probably contributed to the different outcomes. CRuz et al. [28] studied patients with better preserved functional exercise capacity and pulmonary function, while the subjects recruited by BurTin et al. [27] were more severely impaired. The timing of the combined intervention might not have been ideal for these more severely impaired patients. We have observed that during rehabilitation patients already substantially increase their physical activity levels by participating in the programme itself [29]. Patients therefore find it difficult to further increase their participation in daily activities at home while participating in the rehabilitation programme. In a subgroup analysis of a randomised controlled trial, Altenburg et al. [30] also observed that a physical activity counselling intervention offered during a rehabilitation programme resulted in less improvement than counselling offered as a standalone intervention in less impaired patients. Based on these observations future studies should focus on integrating physical activity coaching elements into the final phase of the 
rehabilitation programme. It also seems crucial to continue these coaching interventions after participation in the pulmonary rehabilitation programme has ended and patients return to their home environment.

In order to capture all dimensions of physical activity during these interventions it has been recommended to combine the objective assessment of physical activities with a short and validated patient-reported outcomes questionnaire focusing on the difficulties of performing physical activities related to symptoms [1]. It has become evident that, besides functional capacity and symptoms experienced during activity, behavioural and environmental factors are also related to participation in daily physical activity in patients with COPD [13]. Known correlates and potential barriers to participation in physical activity, such as environmental factors (e.g. seasonal variations, daylight time and availability of suitable infrastructure to engage in physical activity) and behavioural factors (e.g. specific motivation to engage in physical activities outside of the supervised exercise intervention and health beliefs about the potential benefits of engaging in these daily activities), should be registered during intervention studies. The complex interplay between physical, environmental and behavioural factors during interventions aimed at modifying physical activity behaviour is nicely illustrated in a study by Altenburg et al. [30]. They offered an identical physical activity counselling intervention to three different patient populations. While the intervention was effective as a stand-alone therapy in patients with moderate impairments in functional exercise capacity it was less effective when offered in combination with a rehabilitation programme in more physically limited patients [30]. These latter patients were most likely perceiving more difficulties in engaging in physical activity, which might explain part of the differences in response. Unfortunately difficulties in engaging in physical activities were not directly assessed. In contrast to what would be expected, the intervention was however also less effective in a third group of patients with better preserved functional exercise capacity. These patients would be expected to perceive fewer difficulties in engaging in physical activity. It might be that motivational factors or health beliefs (which were unfortunately also not directly assessed) were related to the smaller effects observed in this group.

In summary, strategies to modify physical activity behaviour are gaining popularity in the COPD population. We agree with the conclusion of MANTOANI et al. [18] that coaching interventions and longer lasting rehabilitation programmes have the greatest potential to modify physical activity behaviour. Self-monitoring of daily activities, coaching and goal setting based on relative increases in baseline background activity, with feedback from a pedometer, have been shown to be key components of physical activity counselling interventions. In patients referred to rehabilitation programmes more research is needed on the effectiveness of coaching interventions that are continued after completion of the supervised exercise programme. Capturing different dimensions of physical activity by using recently developed patient-reported outcomes measures is recommended for future studies. Assessment of relevant behavioural and environmental factors related to participation in physical activity will also increase our understanding of mechanisms that can explain differences in treatment responses. Results of these future studies will hopefully be helpful in designing optimal interventions for improving participation in physical activity in patients with COPD.

\section{References}

1 Gimeno-Santos E, Raste Y, Demeyer H, et al. The PROactive instruments to measure physical activity in patients with chronic obstructive pulmonary disease. Eur Respir J 2015; 46: 988-1000.

2 Watz H, Pitta F, Rochester CL, et al. An official European Respiratory Society statement on physical activity in COPD. Eur Respir J 2014; 44: 1521-1537.

3 Lee IM, Shiroma EJ, Lobelo F, et al. Effect of physical inactivity on major non-communicable diseases worldwide: an analysis of burden of disease and life expectancy. Lancet 2012; 380: 219-229.

4 Garber CE, Blissmer B, Deschenes MR, et al. American College of Sports Medicine position stand. Quantity and quality of exercise for developing and maintaining cardiorespiratory, musculoskeletal, and neuromotor fitness in apparently healthy adults: guidance for prescribing exercise. Med Sci Sports Exerc 2011; 43: 1334-1359.

5 Nelson ME, Rejeski WJ, Blair SN, et al. Physical activity and public health in older adults: recommendation from the American College of Sports Medicine and the American Heart Association. Circulation 2007; 116: 1094-1105.

6 DeFina LF, Haskell WL, Willis BL, et al. Physical activity versus cardiorespiratory fitness: two (partly) distinct components of cardiovascular health? Prog Cardiovasc Dis 2015; 57: 324-329.

7 Pitta F, Troosters T, Spruit MA, et al. Characteristics of physical activities in daily life in chronic obstructive pulmonary disease. Am J Respir Crit Care Med 2005; 171: 972-977.

8 Vorrink SN, Kort HS, Troosters T, et al. Level of daily physical activity in individuals with COPD compared with healthy controls. Respir Res 2011; 12: 33.

9 Shrikrishna D, Patel M, Tanner RJ, et al. Quadriceps wasting and physical inactivity in patients with COPD. Eur Respir J 2012; 40: 1115-1122.

10 Troosters T, Sciurba F, Battaglia S, et al. Physical inactivity in patients with COPD, a controlled multi-center pilot-study. Respir Med 2010; 104: 1005-1011.

11 Van Remoortel H, Hornikx M, Demeyer H, et al. Daily physical activity in subjects with newly diagnosed COPD. Thorax 2013; 68: 962-963.

12 Arne M, Janson C, Janson S, et al. Physical activity and quality of life in subjects with chronic disease: chronic obstructive pulmonary disease compared with rheumatoid arthritis and diabetes mellitus. Scand J Prim Health Care 2009; 27: 141-147.

13 Gimeno-Santos E, Frei A, Steurer-Stey C, et al. Determinants and outcomes of physical activity in patients with COPD: a systematic review. Thorax 2014; 69: 731-739. 
14 Van Remoortel H, Hornikx M, Langer D, et al. Risk factors and comorbidities in the preclinical stages of chronic obstructive pulmonary disease. Am J Respir Crit Care Med 2014; 189: 30-38.

15 Waschki B, Kirsten AM, Holz O, et al. Disease progression and changes in physical activity in patients with chronic obstructive pulmonary disease. Am J Respir Crit Care Med 2015; 192: 295-306.

16 Esteban C, Arostegui I, Aburto M, et al. Influence of changes in physical activity on frequency of hospitalization in chronic obstructive pulmonary disease. Respirology 2014; 19: 330-338.

17 Vaes AW, Garcia-Aymerich J, Marott JL, et al. Changes in physical activity and all-cause mortality in COPD. Eur Respir J 2014; 44: 1199-1209.

18 Mantoani LC, Rubio N, McKinstry B, et al. Interventions to modify physical activity in patients with COPD: a systematic review. Eur Respir J 2016; 48: 69-81.

19 Fleig L, Lippke S, Pomp S, et al. Exercise maintenance after rehabilitation: how experience can make a difference. Psychol Sport Exerc 2011; 12: 293-299.

20 Biddle SJ, Brehm W, Verheijden M, et al. Population physical activity behaviour change: a review for the European College of Sport Science. Eur J Sport Sci 2011; 12: 367-383.

21 Greaves CJ, Sheppard KE, Abraham C, et al. Systematic review of reviews of intervention components associated with increased effectiveness in dietary and physical activity interventions. BMC Public Health 2011; 11: 119.

22 Conn VS, Hafdahl AR, Brown SA, et al. Meta-analysis of patient education interventions to increase physical activity among chronically ill adults. Patient Educ Couns 2008; 70: 157-172.

23 Artinian NT, Fletcher GF, Mozaffarian D, et al. Interventions to promote physical activity and dietary lifestyle changes for cardiovascular risk factor reduction in adults: a scientific statement from the American Heart Association. Circulation 2010; 122: 406-441.

24 Bravata DM, Smith-Spangler C, Sundaram V, et al. Using pedometers to increase physical activity and improve health: a systematic review. JAMA 2007; 298: 2296-2304.

25 Loeckx M, Osadnik C, Demeyer H, et al. The likelihood of improving physical activity increases with better functional exercise tolerance in COPD. Eur Respir J 2015; 46: Suppl. 59, OA474.

26 Hornikx M, Demeyer H, Camillo CA, et al. The effects of a physical activity counseling program after an exacerbation in patients with chronic obstructive pulmonary disease: a randomized controlled pilot study. BMC Pulm Med 2015; 15: 136.

27 Burtin C, Langer D, van Remoortel $\mathrm{H}$, et al. Physical activity counselling during pulmonary rehabilitation in patients with COPD: a randomised controlled trial. PLoS One 2015; 10: e0144989.

28 Cruz J, Brooks D, Marques A. Walk2Bactive: a randomised controlled trial of a physical activity-focused behavioural intervention beyond pulmonary rehabilitation in chronic obstructive pulmonary disease. Chron Respir Dis 2016; 13: 57-66.

29 Langer D, Van Remoortel H, Burtin C, et al. Profiles of daily physical activity during three months of pulmonary rehabilitation in patients with COPD. Eur Respir J 2010; 36: Suppl. 54, 331s.

30 Altenburg WA, Hacken NH, Bossenbroek L, et al. Short- and long-term effects of a physical activity counselling programme in COPD: a randomized controlled trial. Respir Med 2015; 109: 112-121. 